\title{
Direct measurements of the magnetic entropy change
}

Nielsen, Kaspar Kirstein; Neves Bez, Henrique; von Moos, Lars; Bjørk, Rasmus; Eriksen, Dan; Bahl, Christian

\section{Published in:}

Review of Scientific Instruments

Link to article, DOI:

$10.1063 / 1.4932308$

Publication date:

2015

Document Version

Peer reviewed version

Link back to DTU Orbit

Citation (APA):

Nielsen, K. K., Neves Bez, H., von Moos, L., Bjørk, R., Eriksen, D., \& Bahl, C. (2015). Direct measurements of the magnetic entropy change. Review of Scientific Instruments, 86(10), [103903].

https://doi.org/10.1063/1.4932308

\section{General rights}

Copyright and moral rights for the publications made accessible in the public portal are retained by the authors and/or other copyright owners and it is a condition of accessing publications that users recognise and abide by the legal requirements associated with these rights.

- Users may download and print one copy of any publication from the public portal for the purpose of private study or research.

- You may not further distribute the material or use it for any profit-making activity or commercial gain

- You may freely distribute the URL identifying the publication in the public portal

If you believe that this document breaches copyright please contact us providing details, and we will remove access to the work immediately and investigate your claim 


\section{Direct measurement of the magnetic entropy change}

Nielsen, Kaspar Kirstein; Neves Bez, Henrique; von Moos, Lars; Bjørk, Rasmus; Eriksen, Dan; Bahl, Christian

Published in:

Review of Scientific Instruments

Publication date:

2015

Document Version

Author final version (often known as postprint)

Link to publication

Citation (APA):

Nielsen, K. K., Neves Bez, H., von Moos, L., Bjørk, R., Eriksen, D., \& Bahl, C. (2015). Direct measurement of the magnetic entropy change. Review of Scientific Instruments.

- You may freely distribute the URL identifying the publication in the public portal ? 


\title{
Direct measurements of the magnetic entropy change
}

\author{
K.K. Nielsen, ${ }^{1}$ a) H.N. Bez, ${ }^{1}$ L. von Moos ${ }^{1}{ }^{1}$ R. Bjørk, ${ }^{1}$ D. Eriksen, ${ }^{1}$ and C.R.H. Bahl ${ }^{1}$ \\ DTU Energy Conversion and Storage, Technical University of Denmark, \\ Frederiksborgvej 399, DK-4000 Roskilde, Denmark
}

(Dated: 22 September 2015)

An experimental device that can accurately measure the magnetic entropy change, $\Delta s$, as a function of temperature, $T$, and magnetic field, $H$, is presented. The magnetic field source is in this case a set of counter-rotating concentric Halbach-type magnets, which produce a highly homogeneous applied field with constant orientation. The field may be varied from 0 to $1.5 \mathrm{~T}$ in a continuous way. The temperature stability of the system is controlled to within $\pm 10 \mathrm{mK}$ and the standard range for the current setup is from $230 \mathrm{~K}$ to $330 \mathrm{~K}$. The device is under high vacuum and we show that thermal losses to the ambient are negligible in terms of the calorimetric determination of the magnetic entropy change, while the losses cannot be ignored when correcting for the actual sample temperature. We apply the device to two different types of samples; one is commercial grade $\mathrm{Gd}$, i.e. a pure second-order phase transition material while the other is $\mathrm{Gd}_{5} \mathrm{Si}_{2} \mathrm{Ge}_{2}$, a first order magnetic phase transition material. We demonstrate the device's ability to fully capture the thermal hysteresis of the latter sample by following an appropriate thermal- and magnetic resetting scheme.

a)kaki@dtu.dk 


\section{INTRODUCTION}

Reliable experimental determination of the magnetocaloric effect (MCE) is crucial for understanding magnetocaloric materials (MCMs), evaluating their possible performance as active refrigerants in a magnetic cooling/heating device and for accurate numerical modeling of active magnetic regenerators $(\mathrm{AMR})^{1}$. The key properties associated with the MCE are the absolute entropy, $s(T, H)$, and magnetization, $M(T, H)$, both as a function of temperature, $T$, and magnetic field, $H$. From the absolute entropy it is possible to derive the

specific heat $c(T, H)=\left.T \frac{\partial s}{\partial T}\right|_{H}$. Obtaining $s(T, H)$ may be done by integrating specific heat measured as a function of $T$ and $H$ or by combining $c(T, H=0)$ with the magnetic entropy change, $\Delta s(T, H)$.

The magnetic entropy change may be found indirectly by applying a Maxwell relation $\partial s / \partial H=\mu_{0} \partial M / \partial T$ where $\mu_{0}$ is the vacuum permeability. It is, however, also possible to measure $\Delta s(T, H)$ in a direct manner using calorimetry ${ }^{2,3}$. This is advantageous as magnetization data usually have low thermal resolution, which will result in a smearing of data when numerical differentiation is performed. Temperature control is usually also more stable in the direct measurement as opposed to in a VSM where inevitably the gas temperature has to be adjusted continuously thus leading to fluctuations. In the direct measurement equipment a relatively large thermal reservoir dampens fluctuations to a small level.

In this paper we describe an experimental setup that has been realized at the Technical University of Denmark for direct measurements of $\Delta s$, which is applied to measure an MCM with a first order magnetic phase transition $\left(\mathrm{Gd}_{5} \mathrm{Si}_{2} \mathrm{Ge}_{2}\right)$ and one with a second order transition (Gd). Through appropriate thermal and magnetic resetting of the sample we demonstrate the ability of the device to probe and capture the hysteretic behavior of $\mathrm{Gd}_{5} \mathrm{Si}_{2} \mathrm{Ge}_{2}$. Our device resembles that of Ref. 2 with the addition that our device has a fully automated systematic resetting capability (both thermal and magnetic reset). Our magnetic field source is furthermore made of permanent magnets rather than an electromagnet and the field change from zero to max $(1.5 \mathrm{~T})$ field can be achieved in a continuous way. 


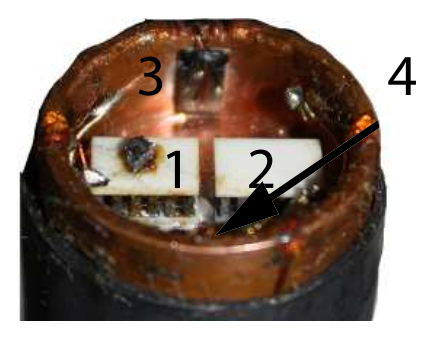

FIG. 1. Picture of the top of the cold finger. The numbers indicate the various components: 1 and 2 are the Peltier elements, 3 the Hall probe magnetic field sensor and 4 the Pt-100 thermo-element. The diameter of the disk is $20 \mathrm{~mm}$.

\section{EXPERIMENTAL SETUP}

A schematic of the experimental setup is shown in Fig. 1. The sample is placed on one of two small $\left(3.25 \times 4.88 \mathrm{~mm}^{2}\right)$ identical Peltier-cells (Optotec OT08), which are glued to a cold finger made of copper. A larger Peltier cell (TEC1-12714S) is located at the bottom of the cold finger. On one side it is connected with thermal grease to the cold finger and on the other side to a heat exchanger, which in turn has a continuous flow of a chilled fluid from an external temperature control bath (Julabo CF40 Cryo-Compact Circulator). By regulating the magnitude and direction of the current through the large Peltier cell, as well as the temperature of the chiller fluid, it is possible to control the temperature of the cold finger. Copper was chosen for its excellent thermal properties (high conductivity and large thermal mass) and because it is diamagnetic.

The temperature range of the system is from $230 \mathrm{~K}$ to $330 \mathrm{~K}$. The temperature of the system is measured in two places by Pt-100 resistance thermometers. One at the bottom of the cold finger and one in the top (number 4 in Fig. 1) very close to the Peltier cells. Here, a Hall-probe (Arepoc HHP-NU), number 3 in Fig. 1, for measuring the magnetic field is also installed.

The cold-finger is situated in a vacuum chamber connected to a turbo-pump, giving a typical operational pressure of approximately $3 \times 10^{-6}$ mbar. The larger heater Peltier cell is controlled via a power supply unit controlled by a PC. A PID control program implemented in LabView and using the temperature measured by the bottom temperature sensor as the process parameter, controls the current delivered to the Peltier cell and thus the temperature of the cold-finger. The voltage measurements are done using a Keithley 2700 

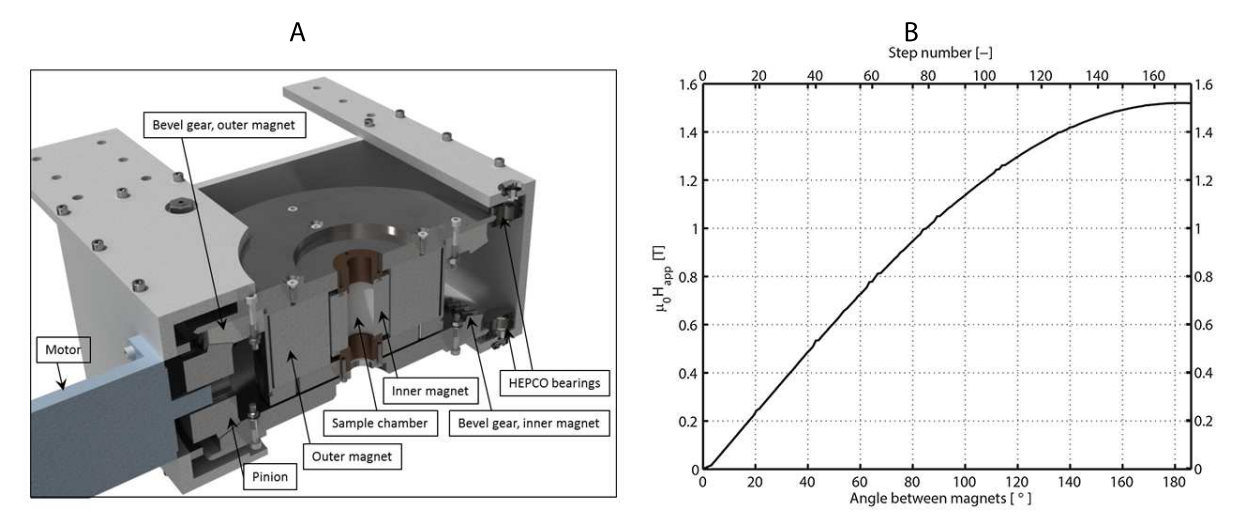

FIG. 2. A: Cross sectional view of the mechanical setup for support and counter rotation of the concentric Halbach magnets. B: The generated magnetic field as a function of the angle of rotation between the two concentric Halbach cylinders. Note that when the angle between the magnets is changed from zero to $180^{\circ}$ this corresponds to a rotation of each magnet of $90^{\circ}$.

Digital MultiMeter with a 20 channel Keithley 7700 board except for the Hall-probe, which is measured with a USB2AD DAQ module from Arepoc ${ }^{4}$.

The magnetic field applied to the setup is provided by a rotating Halbach cylinder permanent magnet configuration. This exact permanent magnet assembly is described in detail elsewhere $^{5}$. The generated magnetic field is controlled by counter-rotating two concentric Halbach cylinders with equal angular velocity magnitude. Consequently, the direction of the field in the sample chamber is constant. The two Halbach cylinders are mechanically counter-rotated by the setup shown in Figure 2A. A Powerpac stepper motor (N33HRLJLNK-NS-00) with a Technosoft controller (IDM240-5EI) is used for turning a pinion connected with two bevel gears of equal diameters, one fixed to the inner magnet, the other to the outer magnet. The gears and thus the magnets are supported on the outside by HEPCO bearings. The field as a function of the angle between the magnets is shown in Fig. 2B. As can clearly be seen from the figure, the field can be adjusted from 0 to $1.5 \mathrm{~T}$ continuously.

Normally the field is varied at $0.05 \mathrm{~T} / \mathrm{s}$. Test were done using an aluminum dummy sample to detect any eddy current induced, but within the noise of the signal nothing was observed. 


\section{A. Measurement of magnetic entropy change}

A temperature difference, $\Delta T$, between the top and bottom of one of the Peltier cells on the cold finger will result in a voltage signal, $U$, due to the Seebeck effect:

$$
U=-S \Delta T
$$

with the Seebeck coefficient, $S$, being specific for the Peltier cells and generally varying as a function of temperature. The heat flux corresponding to the temperature difference is

$$
\dot{Q}=\kappa \Delta T=-\frac{\kappa}{S} \Delta U
$$

with the conductance of the Peltier cell $\kappa=1 / R . \quad R$ is found as $R=\Delta T_{\max } / \dot{Q}_{\max }=$ $67 \mathrm{~K} / 0.6 \mathrm{~W}=112 \mathrm{KW}^{-1}$. This is, however, merely stated at room temperature. Here, the differential signal is used, i.e. the difference in signal between the Peltier cell with a sample on it and the empty Peltier cell $\left(\Delta U=U_{\text {sample }}-U_{\text {empty }}\right)$.

For measurements of the magnetic entropy change the system is at a fixed temperature and the applied magnetic field is varied from a starting value, $H_{0}$, to a final value, $H_{1}$. The response of the difference in voltage signal of the two Peltier cells (see Fig. 3A for an example) is then integrated in time, which yields:

$$
\Delta s\left(T, H_{0}, H_{1}\right)=\frac{q}{T}=\frac{Q}{T m_{\mathrm{s}}}=\frac{\kappa}{S T m_{\mathrm{s}}} \int_{t_{0}}^{t_{1}} \Delta U \mathrm{~d} t
$$

where the mass of the sample is denoted $m_{\mathrm{s}}$. The voltage signal is integrated in time, $t$, from the start of the signal $t_{0}$ to the time $t_{1}$ where the voltage difference has returned to zero. The change in heat per mass is $q$ while the total change in heat of the sample is $Q$. The value $\Delta s\left(T, H_{0}, H_{1}\right)$ is thus the magnetic entropy change upon the field change from $H_{0}$ to $H_{1}$ at the temperature $T$.

\section{B. Calibration}

In order to obtain a proper conversion from the Peltier voltage signal to a heat flux, the signals must be calibrated. One method for calibrating the system is to use a sample with a known heat capacity, e.g. pure $\mathrm{Cu}^{6}$. For temperature values outside the range given in Ref. 6, i.e. from $300 \mathrm{~K}$ to $320 \mathrm{~K}$, the values are linearly extrapolated. Assuming a constant 

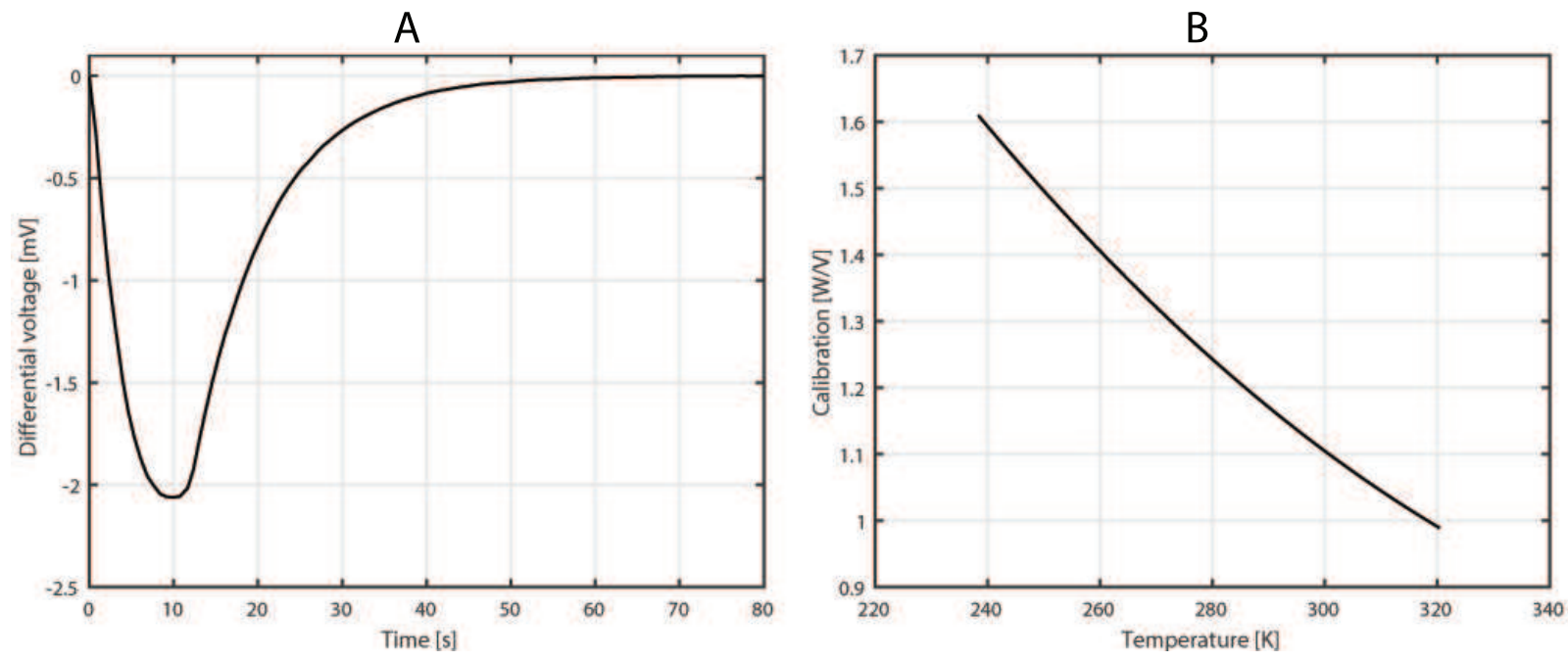

FIG. 3. A: The differential voltage signal as a function of time of a $38.9 \mathrm{mg}$ sample of commercial grade Gd. The applied field is changed from $0.01 \mathrm{~T}$ to $1.26 \mathrm{~T}$ with a ramp rate of $0.1 \mathrm{~T} / \mathrm{s}$. The time axis is zero at the time $t_{0}$. B: The calibration of the Peltier signal $(\kappa / S$ in Eq. 4$)$ from a measurement of a pure $\mathrm{Cu}$ sample.

temperature rate $\dot{T}$ (i.e. the system is set to ramp the temperature), one obtains:

$$
\begin{aligned}
& \dot{Q}=c_{\mathrm{ref}} m_{\mathrm{ref}} \dot{T} \Rightarrow \\
& \frac{\kappa}{S}=-\frac{c_{\mathrm{ref}} m_{\mathrm{ref}} \dot{T}}{\Delta U},
\end{aligned}
$$

where the mass of the reference $\mathrm{Cu}$ sample is denoted $m_{\text {ref }}$ and the specific heat of the reference sample is $c_{\text {ref }}$. The calibration is plotted in Fig. $3 \mathrm{~B}$.

As the sample temperature is not directly measured it is necessary to estimate the temperature difference between the sample on top of the Peltier cell and the Pt-100 element below it. It is assumed that the thermal resistance of the $\mathrm{Cu}$ is negligible and that it is $R=112 \mathrm{KW}^{-1}$ for the measurement Peltier cell, as found above. Furthermore, the highvacuum effectively means that the only mechanism for losses from the sample to the ambient is through radiation, i.e.

$$
\dot{Q}_{\text {loss }}=\alpha A_{\mathrm{s}}\left(T_{\mathrm{amb}}^{4}-T_{\mathrm{s}}^{4}\right)
$$

where $\alpha$ is Stefan-Boltzmann's constant, $A_{\mathrm{s}}$ the radiating surface area of the sample, $T_{\mathrm{amb}}$ the ambient temperature and $T_{\mathrm{s}}$ the sample temperature. Here, the emissivity of the sample is assumed to be 1. In steady-state the heat loss from the sample must be matched by the 


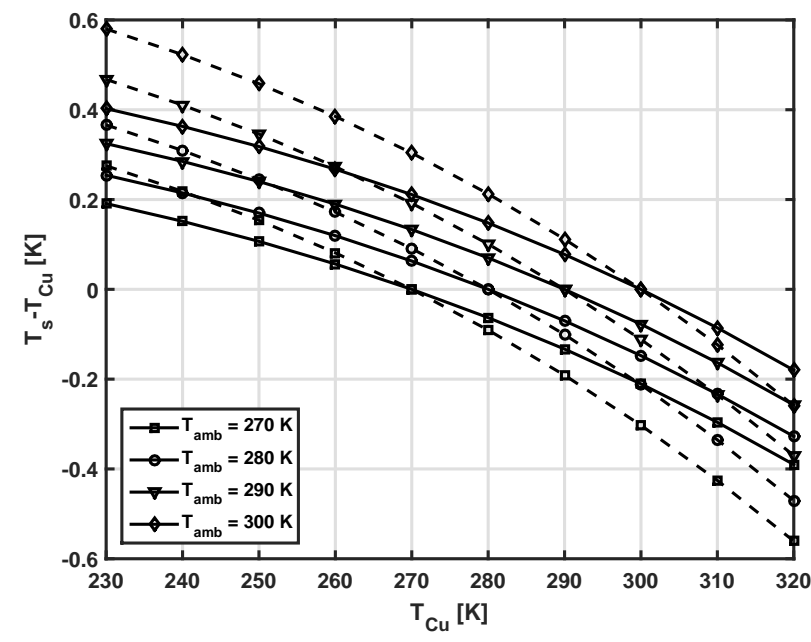

FIG. 4. Temperature difference between sample and $\mathrm{Cu}$ cold finger as a function of the cold finger temperature for different ambient temperatures. Solid lines are based on Eq. 6 while dashed lines are based on a detailed 3D model of the system solved using Comsol Multiphysics. The sample area is assumed equal to the Peltier surface area, $A_{\mathrm{s}}=15.86 \mathrm{~mm}^{2}$.

temperature difference between the sample and the top of the $\mathrm{Cu}$ cold finger, i.e.:

$$
\Delta T_{\mathrm{s}}=T_{\mathrm{s}}-T_{\mathrm{Cu}}=\dot{Q}_{\mathrm{loss}} R
$$

Combining Eqs. 5 and 6 results in a fourth order polynomial in $T_{\mathrm{s}}$, where the roots are easily found numerically.

Since the system is not completely symmetric, the temperature difference between sample and the $\mathrm{Cu}$ cold finger was also found through a 3D model in Comsol multiphysics with radiation losses enabled. Figure 4 shows the difference in temperature between the sample and the cold finger as a function of the cold finger temperature for various ambient temperatures. The simple model, represented by Eqs. 5-6, is seen to adequately catch the temperature difference compared to the more detailed Comsol model within $0.2 \mathrm{~K}$. It is thus straight-forward to adjust for the thermal resistance between the Pt-100 element and the sample in order to obtain the correct sample temperature.

\section{Measurement uncertainties}

In order to evaluate the total measurement error the following assumptions are made:

1. Uncertainties in measurement of the magnetic field and time are negligible. 


\section{Possible co-variance is ignored.}

The total error in the magnetic entropy change can be found by considering equation 3. This equation can be split into two parts: the integral part, $X(t)_{T}=\Delta U(t)$, and the calibration part, $\left(C=\kappa /\left(S T m_{s}\right)\right)$. The total error is given by:

$$
\left(\frac{\sigma(\Delta s)}{|(\Delta s)|}\right)^{2}=\left(\frac{\sigma(C)}{|C|}\right)^{2}+\left(\frac{\sigma(A)}{|A|}\right)^{2}
$$

where the first term on the right hand side is the relative error of the calibration term, while the second term is the relative error of the integral part.

The relative error sum for the calibration term is given by:

$$
\frac{\sigma(C)}{|C|}=\sqrt{\left(\frac{\sigma\left(c_{\mathrm{ref}}\right)}{\left|c_{\mathrm{ref}}\right|}\right)^{2}+\left(\frac{\sigma\left(m_{\mathrm{ref}}\right)}{\left|m_{\mathrm{ref}}\right|}\right)^{2}+\left(\frac{\sigma(\dot{T})}{|\dot{T}|}\right)^{2}+\left(\frac{\sigma(T)}{|T|}\right)^{2}+\left(\frac{\sigma\left(m_{\mathrm{s}}\right)}{\left|m_{\mathrm{s}}\right|}\right)^{2}}
$$

The standard deviation of the voltage difference $(\sigma(\Delta U))$ was calculated based on the steady-state voltage difference signal. Over the applicable temperature range the standard deviation of the voltage difference was found not to be a function of temperature. The standard deviation of the integral $A(T, t)=\int \Delta U d t$ is found through:

$$
\sigma(A(T))=\left(\sum_{n=1}^{N}\left[\left(\Delta t_{n}\right)^{2}(\sigma(\Delta U(T)))^{2}\right]\right)^{1 / 2}
$$

where the sum is over $N$ datapoints and $\Delta t_{n}$ is the difference in time between two adjacent datapoints. Figure 5 shows the two main relative uncertainties that comprise the uncertainty of $\Delta s$. It is observed that the relative error in the integral part (Fig. 5A) is small compared to the calibration part (Fig. 5B). Furthermore, it is seen that for $T \gg T_{\mathrm{C}}$ and $T \ll T_{\mathrm{C}}$ the error of the integral term increases. This behaviour is due to the fact that at $T_{\mathrm{C}}$ the area of $\Delta U(t)$ peak is relatively bigger than at other temperatures. Finally, the relative error of the calibration term decreases as the temperature increases due to the increasing voltage difference with temperature. Figure 6 shows the magnetic entropy change of Gd as a function of temperature for an applied field change of $1.0 \mathrm{~T}$ with the associated errorbars calculated from Eq. 7.

Pecharsky and Gschneidner Jr. ${ }^{7}$ systematically calculated the error propagation on $\Delta s$ based on $c_{p}$ and magnetization data. It was shown that for $\mathrm{Gd}$ the errors at $5 \mathrm{~T}$ can be between 20-30\% for both methods, which is significantly higher than the $7.5 \%$ (with a $\left.\mu_{0} \Delta H=1.0 \mathrm{~T}\right)$ in the equipment presented here. It is noted that this error will decrease further as the field change is increased. 

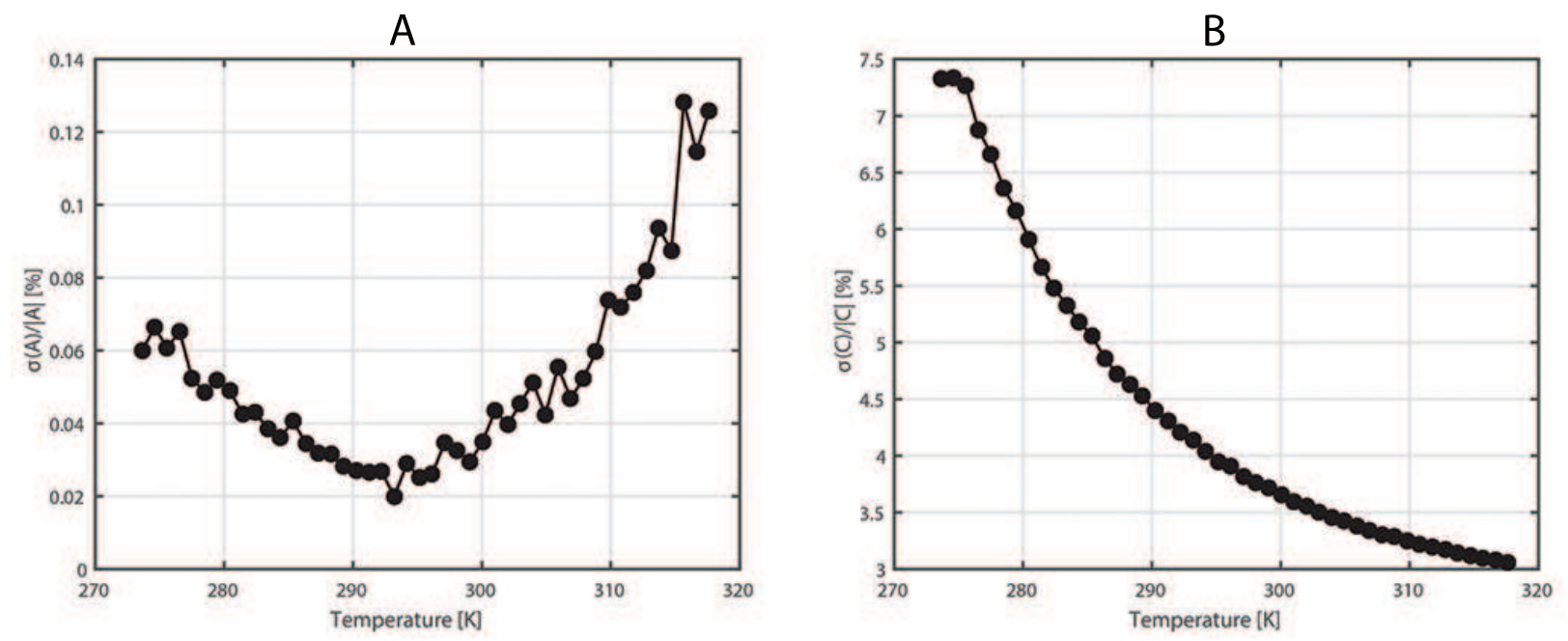

FIG. 5. A: The relative standard deviation of the integral term. B: the calibration term as a function of temperature. A minimum in $\sigma(A) /|A|$ at $T_{\mathrm{C}} \approx 292 \mathrm{~K}$ region is seen.

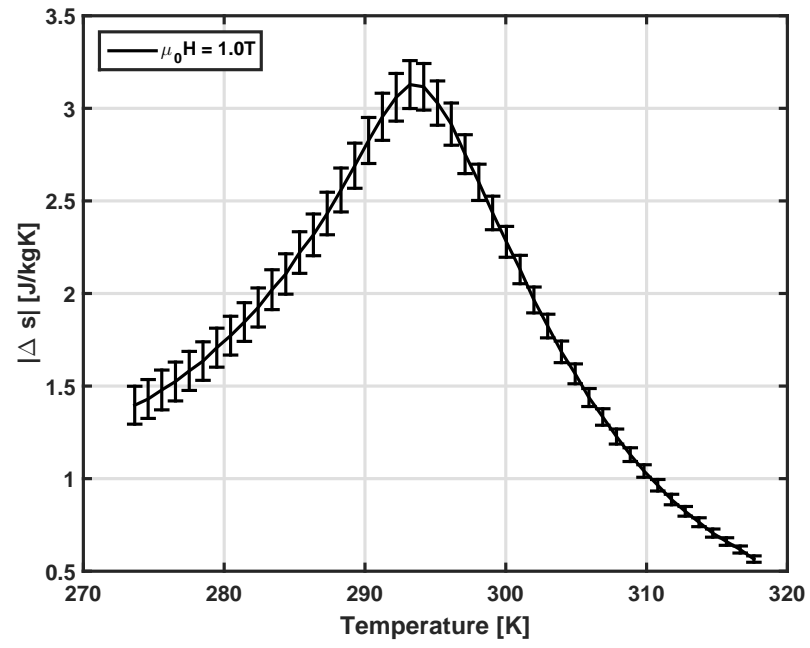

FIG. 6. Magnetic entropy change of $\mathrm{Gd}$, for $\mu_{0} \Delta H_{\text {app }}=1.0 \mathrm{~T}$, as a function of the temperature and the associated error of the measurement. Note that the field considered is the applied magnetic field.

\section{EXAMPLES OF ENTROPY MEASUREMENTS}

A polycrystalline commercial grade Gd sample $\left(38.9 \mathrm{mg}, 0.9 \times 2.3 \times 2.9 \mathrm{~mm}^{3}\right)$ was mounted on the measurement Peltier cell and measured in the temperature range from $275 \mathrm{~K}$ to 315 K. At each temperature the sample was measured in 5 different applied fields, in the range from $0.18 \mathrm{~T}$ to $1.50 \mathrm{~T}$, by applying the first field, removing it again and then applying the 
second field etc. For each field application, a signal similar to that in Fig. 3A is measured and integrated to give the magnetic entropy change for the current temperature and field change, using Eq. 3. The results of the measurements are shown in Fig. 7, where they are compared to measurements done on the same sample, using a LakeShore 7407 Vibrating Sample Magnetometer (VSM) to obtain the magnetic entropy change indirectly through the Maxwell relation ${ }^{8}$. The same orientation of the sample in the two measurements with respect to the applied field was prohibited by the sample geometry. In order to make a proper correction for the differing demagnetizing fields in the two cases the following procedure was followed: The average internal field in the sample while measuring in the VSM, $H_{\mathrm{VSM}}$, was found as a function of temperature and applied field using the appropriate demagnetization factor $\left(N_{\mathrm{VSM}}=0.18\right.$, assuming a rectangular sample $\left.{ }^{9}\right)$, i.e. through the direct calculation $H_{\mathrm{VSM}}=H_{\mathrm{app}, \mathrm{VSM}}-N_{\mathrm{VSM}} M$. This directly gives the average magnetization, $M$, of the sample as a function of temperature and internal field. In order to find the internal field in the calorimetric measurements, $H_{\Delta s}$, the average demagnetization factor $N_{\Delta s}=0.26$ (again assuming a rectangular prism ${ }^{9}$ ) was used in $H_{\Delta s}=H_{\text {app }, \Delta s}-N_{\Delta s} M$, which is solved iteratively using $M$ from the VSM measurements. For each applied field in the calorimeter the internal field in the sample is thus found as a function of temperature. Finally, this field is used to interpolate in the calculated entropy change data from the VSM measurements. The magnetic entropy change is given as a function of temperature for various applied fields (in the calorimeter) while the VSM data has been interpolated as described above so that the two sets of measurements have the same internal field in each data point. The direct method for measuring the entropy change agrees quite well with the results from the indirect conventional approach, although with a slight tendency for the direct measurements to produce a sharper peak. This may in part be explained from the fact that the VSM-based entropy change data is calculated through the temperature-derivative of the magnetization, which in turn will tend to round the peak slightly. Still, both results are inside each others uncertainties.

\section{A. Thermal resetting and hysteresis measurements}

Both thermal and magnetic hysteresis are present to some degree in MCMs with a first order transition. In order to measure this accurately it is crucial to be able to accurately 


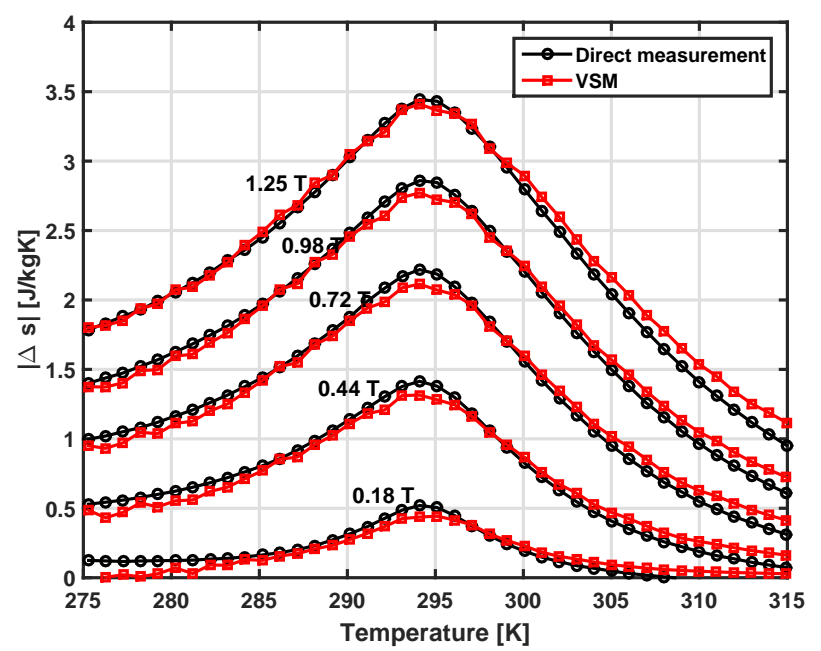

FIG. 7. The magnetic entropy change of Gd found using the appropriate Maxwell relation on VSM magnetization data and through the direct calorimetric approach described in this paper. The applied magnetic fields of the direct measurements is indicated in the figure. The VSM data has been interpolated in order to correct for demagnetization according to the method described in the text. The mass of the sample was $38.9 \mathrm{mg}$

control the state and history of the sample. One way to do this is to reset the sample between each measurement. This means that after temperature equilibration and subsequent magnetization the sample is cooled or heated to a temperature outside the hysteresis region. The excellent temperature and magnetic field control offered by the experimental setup described here enables hysteresis measurements to be done in an automated, efficient and accurate way. Specifically it is important to note that the instrument does not overshoot when changing the temperature or field. Such overshooting is quite common in measurement devices, such as VSMs, and will make the characterisation and interpretation of hysteresis difficult. The average thermal equilibrium time for stabilizing at a given temperature is approximately four minutes.

The experiment can be set to automatically do a thermal reset by going sufficiently above or below $T_{\mathrm{C}}$ between each measurement. If the sample is not reset it may enter a meta-stable state ${ }^{10}$. A polycrystalline bulk sample of the well known and highly hysteretic magnetocaloric material $\mathrm{Gd}_{5} \mathrm{Si}_{2} \mathrm{Ge}_{2}$, with $T_{\mathrm{C}}$ around $272 \mathrm{~K}$, provided by Ames Laboratory, was measured in five different protocols applied to each temperature point:

1. No reset and field change from 0 to $1.5 \mathrm{~T}$ (called "Non-equilibrium" in Fig. 8) 
A

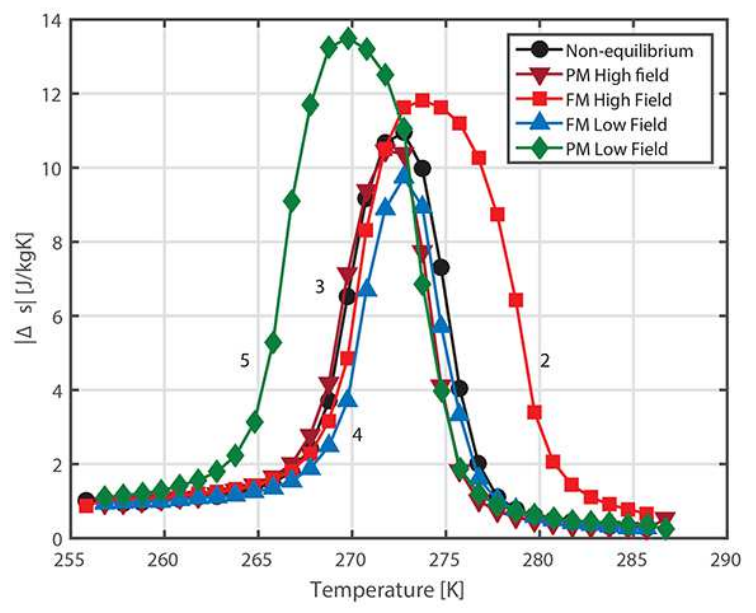

B

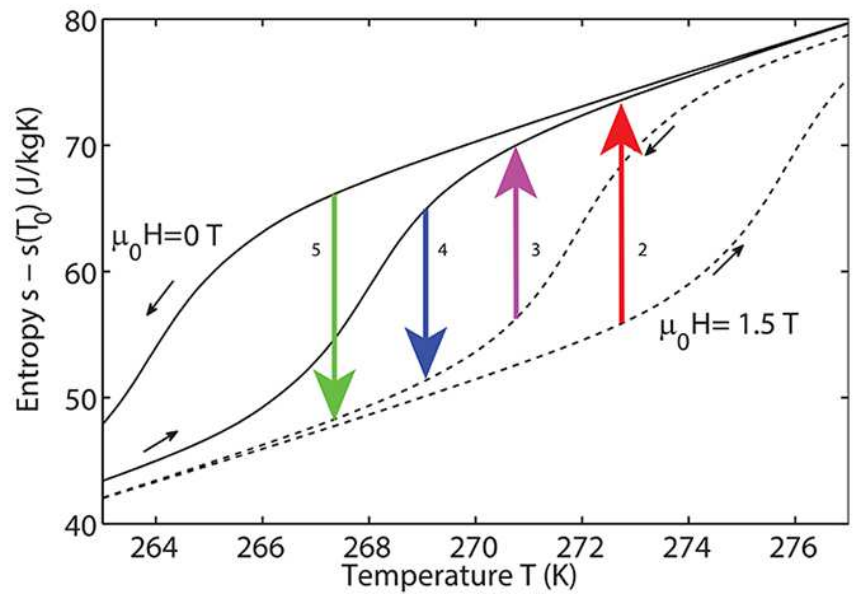

FIG. 8. A: The magnetic entropy change as a function of temperature for the polycrystalline sample $\mathrm{Gd}_{5} \mathrm{Si}_{2} \mathrm{Ge}_{2}$ under an applied field change from zero to $1.5 \mathrm{~T}$. The sample was measured under five different conditions as described in the text. B: the entropy diagram derived from specific heat data (see Ref. 11). The arrows indicate the different experiment modes (at random temperatures). The curves/arrows in the two figures are numbered according to the definitions in the text. The mass of the sample was $10.2 \mathrm{mg}$.

2. Ferromagnetic reset (at $T=233 \mathrm{~K}$ ) and field change from 1.5 to $0 \mathrm{~T}$ (called "FM High Field" in Fig. 8)

3. Paramagnetic reset (at $T=293 \mathrm{~K}$ ) and field change from 1.5 to $0 \mathrm{~T}$ (called "PM High Field" in Fig. 8)

4. Ferromagnetic reset (at $T=233 \mathrm{~K}$ ) and field change from 0 to $1.5 \mathrm{~T}$ (called "FM Low Field" in Fig. 8)

5. Paramagnetic reset (at $T=293 \mathrm{~K}$ ) and field change from 0 to $1.5 \mathrm{~T}$ (called "PM Low Field" in Fig. 8)

The non-equilibrium, ferromagnetic low to high field and paramagnetic high to low field data align fairly well, while the ferromagnetic high to low field and paramagnetic low to high field are significantly different. This is expected due to the hysteresis of the sample (see, e.g., Ref. 11 for details). Figure 8B conceptually shows how the different curves appear. Once a magnetization has been done the sample is considered in a non-equilibrium state and 
in order to accurately predict the outcome of successive magnetizations / demagnetizations without a thermal reset, modeling is needed ${ }^{11}$. It is debatable whether the first measurement after a given resetting procedure results in the magnetic entropy change only. As we have shown, subsequent (de-)magnetizations result in self-consistent data. However, the external driving mechanism for the experiment is a change in applied magnetic field and from that perspective the resulting measurement is the magnetic entropy change.

\section{CONCLUSION}

A custom device for direct measurement of the magnetic entropy change was presented and examples of measurements of two different types of samples were shown. The measurements agree well with data obtained through conventional magnetization measurements. Also, the thermal hysteresis in materials with a first order transition can be full characterized due to the feature of thermal resetting available in the device. The high accuracy with which the temperature and applied field can be controlled and the fact that losses to the ambient are virtually negligible (in terms of the calorimetry; they have to be taken into account when determining the sample temperature) enables high resolution and accuracy measurements.

\section{ACKNOWLEDGEMENTS}

This work was in part financed by the ENOVHEAT project which is funded by Innovation Fund Denmark (contract no 12-132673). We also thank Professors K.A. Gschneidner Jr. and V. Pecharsky from Ames Laboratory for providing the $\mathrm{Gd}_{5} \mathrm{Si}_{2} \mathrm{Ge}_{2}$ sample.

\section{REFERENCES}

${ }^{1}$ K. K. Nielsen, J. Tusek, K. Engelbrecht, S. Schopfer, A. Kitanovski, C. R. H. Bahl, A. Smith, N. Pryds, A. Poredos, Review on numerical modeling of active magnetic regenerators for room temperature applications, Int. J. Refrig. 34 (2011) 603-616.

${ }^{2}$ V. Basso, C. P. Sasso, M. Küpferling, A Peltier cells differential calorimeter with kinetic correction for the measurement of $\mathrm{c}(\mathrm{p})(\mathrm{H}, \mathrm{T})$ and Delta $\mathrm{s}(\mathrm{H}, \mathrm{T})$ of magnetocaloric materials, Rev. Sci. Instrum. 81 (2010) 113904. 
${ }^{3}$ M. Kuepferling, C. P. Sasso, V. Basso, L. Giudici, An isothermal peltier cell calorimeter for measuring the magnetocaloric effect, IEEE Trans. Magn. 43 (2007) 2764-2766. doi: 10.1109/TMAG. 2007.893862 .

${ }^{4}$ www.arepoc.sk, Arepoc s.r.o., bratislava, Slovakia.

${ }^{5}$ R. Bjørk, C. R. H. Bahl, A. Smith, N. Pryds, Comparison of adjustable permanent magnetic field sources, J. Magn. Magn. Mater. 322 (2010) 3664-3671.

${ }^{6}$ G. T. Furukawa, W. G. Saba, M. L. Reilly, Critical analysis of the heat capacity data of the literature and evaluation of thermodynamic properties of copper, silver and gold from 0 to $300 \mathrm{k}$, Tech. rep., NIST (1968).

${ }^{7}$ V. K. Pecharsky, K. A. Gschneidner Jr., Magnetocaloric effect from indirect measurements: Magnetization and heat capacity, J. Appl. Phys. 86 (1999) 565-575.

${ }^{8}$ R. Bjørk, C. R. H. Bahl, M. Katter, Magnetocaloric properties of $\mathrm{LaFe}_{13-x-y} \mathrm{Co}_{x} \mathrm{Si}_{y}$ and commercial grade Gd, J. Magn. Magn. Mater. 322 (2010) 3882-3888.

${ }^{9}$ A. Aharoni, Demagnetizing factors for rectangular ferromagnetic prisms, J. Appl. Phys. 83 (1998) 3432-3434.

${ }^{10}$ L. Caron, Z. Q. Ou, T. T. Nguyen, D. T. Cam Thanh, O. Tegus, E. Brück, On the determination of the magnetic entropy change in materials with first-order transitions, J. Magn. Magn. Mater. 321 (2009) 3559-3566.

${ }^{11}$ L. von Moos, C. Bahl, K. Nielsen, K. Engelbrecht, The influence of hysteresis on the determination of the magnetocaloric effect in $\mathrm{Gd}_{5} \mathrm{Si}_{2} \mathrm{Ge}_{2}$, Journal of Physics D: Applied Physics 48 (2) (2015) 025005. 